\title{
Restabelecimento do sorriso com restaurações em resina composta após clareamento dental: relato de caso
}

\author{
Smile reestablishment with resin composite \\ restorations after tooth bleaching: case report
}

\author{
Madelei Jomhara Vargas-Mendoza' (1) \\ Adriana Maria Ribeiro Junqueira ${ }^{2}$ (1) \\ Claudia Cristina da Costa Telles ${ }^{3}$ \\ Giovana Rodrigues Perin Carnaúba 4 (c) \\ Waldemir Francisco Vieira-Junior ${ }^{5}$ (1)
}

\begin{abstract}
${ }^{1-4}$ Faculdade de Medicina e Odontologia São Leopoldo Mandic (Campinas). São Paulo, Brasil. vm24292@gmail.com, adriana.arruda@sImandic.edu.br, pitelles@bol.com.br, giopercd@hotmail.com ${ }^{5}$ Autor para correspondência. Faculdade de Medicina e Odontologia São Leopoldo Mandic (Campinas). São Paulo, Brasil. waldemir.junior@slmandic.edu.br
\end{abstract}

RESUMO | INTRODUÇÃO: Atualmente, os pacientes almejam um sorriso harmônico e natural, e procedimentos minimamente invasivos devem ser priorizados. Dentre esses, o clareamento dental associado às restaurações diretas com resina composta são tratamentos indicados para alteração de cor e forma dental, devolvendo estética e função. OBJETIVO: O presente trabalho tem como objetivo apresentar, através de um relato de caso clínico, o restabelecimento de um sorriso possibilitado pelo clareamento dental e restaurações diretas em resina composta. RELATO DE CASO: Paciente do sexo feminino, 33 anos, compareceu em consulta clínica se queixando da estética do seu sorriso após a remoção do aparelho ortodôntico. Os dentes apresentavam alteração de cor e forma, e assim, foram planejados clareamento dental e restaurações diretas em resina composta. O clareamento dental foi realizado pela técnica combinada e após 14 dias, as restaurações em resina composta nos incisivos, caninos e pré-molares superiores foram confeccionadas. CONCLUSÃO: O tratamento clareador e as restaurações em resina composta são abordagens conservadoras que possibilitaram o restabelecimento estético do sorriso.

PALAVRAS-CHAVE: Resinas compostas. Clareamento dental. Clareadores. Estética.

\begin{abstract}
INTRODUCTION: Currently, patients desire a harmonious and natural smile, for which minimally invasive procedures must be prioritized. Teeth bleaching and direct resin composite restorations are indicated treatments for changing tooth color and shape, restoring aesthetics and function. OBJECTIVE: The aim of this study was to present a clinical case showing that a smile can be reestablished through teeth bleaching and direct resin composite restorations. CASE REPORT: A 33-year-old female patient presented at a clinical consultation to discuss the aesthetics of her smile after finishing orthodontic treatment. Her teeth showed altered color and shape, so dental bleaching and direct restorations were planned. Dental bleaching was performed using the combined technique, and 14 days later, composite resin restorations were completed in the maxillary incisors, canines, and premolars. CONCLUSION: Tooth bleaching and resin composite restorations are conservative approaches that allowed the aesthetic reestablishment of one's smile.
\end{abstract}

KEYWORDS: Composite resins. Tooth bleaching. Bleaching agents. Esthetics. 


\section{Introdução}

A aparência estética é uma das preocupações humanas, o que leva, constantemente, à busca dos pacientes por tratamentos com a finalidade de se obter um sorriso natural e harmonioso. Atualmente, os procedimentos restauradores têm contribuído consideravelmente para a resolução de problemas relacionados à estética do sorriso ${ }^{1}$. Dentre os aspectos associados à aparência estética do sorriso, as alterações da cor dental possuem etiologia relacionada a fatores extrínsecos ou intrínsecos, sendo um fator que pode influenciar a atratividade do sorriso²

Dentre os tratamentos para alteração de cor dental, quando corretamente indicado, realizado ou supervisionado por um profissional, o clareamento dental é considerado seguro, eficaz e minimamente invasivo, como demonstrado por relatos de casos e revisões sistemáticas ${ }^{3,4}$. No entanto, uma sensibilidade oriunda do tratamento clareador pode ser esperada devido a difusão de peróxido de hidrogênio e seus subprodutos até a polpa 5 . Protocolos para controle de tal feito adverso devem ser empregados.

Outras técnicas de reabilitação foram sugeridas para solucionar problemas associados ao alinhamento, forma e cor do sorriso, ou ainda para tratar dentes extensamente destruídos. Os tratamentos com coroas cerâmicas ou facetas indiretas são abordagens validadas e se caracterizam pela confecção de preparos no tecido dental, entretanto quando incorretamente indicados ou realizados podem ocasionar desgastes excessivos ou danos aos tecidos circundantes ${ }^{6}$. Considerando o desenvolvimento técnico-científico dos materiais e procedimentos, outra abordagem possível atualmente é a indicação de materiais restauradores diretos, que tem seu uso cada vez mais difundido entre os profissionais devido ao sucesso clínico, boas propriedades das resinas compostas e possibilidade de preservação dos tecidos dentários durante o preparo dental .

A técnica restauradora direta possibilita devolver ao dente restaurado suas propriedades ópticas, assim como corrigir defeitos de forma e contorno. Os resultados estéticos e funcionais desse tratamento envolvem o entendimento do profissional sobre a técnica adesiva e restauradora, propriedades cromáticas do dente e a relação da estrutura natural com os materiais restauradores $\stackrel{乛}{ }$. Baseados nos princípios da Odontologia minimamente invasiva, o clareamento dental e as restaurações diretas em resina composta se apresentam como tratamentos conservadores. Considerando a relevância social e profissional em se difundir técnicas seguras com preservação dos tecidos duros dentais sadios, o objetivo deste trabalho é relatar a possibilidade de restabelecimento de um sorriso funcional, estético e harmonioso por técnicas como o clareamento dental e restaurações diretas com resina composta.

\section{Caso clínico}

Paciente, mulher, 33 anos compareceu à clínica de especialização em Dentística da Faculdade de Odontologia São Leopoldo Mandic (Campinas), queixando-se da estética do seu sorriso após dois anos do fim de seu tratamento ortodôntico, enfatizando sua insatisfação em relação à cor e forma dental. A paciente assinou um termo de consentimento livre esclarecido e o relato de caso foi submetido a avaliação por um comitê de ética em pesquisa (CAAE: 36946020.4.0000.5374). Inicialmente, foi realizada a anamnese, e ao exame clínico e radiográfico, observou-se que a paciente apresentava condição bucal e periodontal satisfatórias. Na avaliação oclusal intraoral, notou-se que apesar de um movimento de lateralidade esquerda adequado, o movimento de lateralidade do lado direito não apresentava guia em canino. Os dentes 11 e 21 apresentavam alterações de forma com ângulos incisais em desarmonia, e o dente 14 apresentava uma fratura de restauração (oclusal e palatal). O aspecto inicial do sorriso está apresentado na Figura 1.

Após a tomada de cor inicial dos dentes (D3, VITA Classical, VITA Zahnfabrik, Alemanha) foi realizada a moldagem do arco superior e inferior com um hidrocolóide irreversível (Alginato Hydrogum 5, Zhermack/ Dentsply Sirona, EUA) e envasamento do molde com gesso especial tipo IV (Durone, Zhermack/Dentsply Sirona, EUA) para obtenção de modelos de estudo. No articulador semi-ajustável e considerando o enceramento (Figura 2A), o planejamento foi realizado baseado nos movimentos de lateralidade, protrusão com 
respeito estético e funcional das guias. Após esclarecimentos sobre as possibilidades diretas e indiretas de tratamento restaurador, a paciente optou pela reabilitação estética e funcional com clareamento dental e restauração direta em resina composta. Como plano de tratamento foi idealizado o clareamento dental pela técnica combinada até os pré-molares de ambos os arcos e as restaurações em resina composta nos dentes $11,21,12,22,13,23,14,24$. A opção por este tratamento foi baseada considerando os princípios de mínima intervenção e máxima conservação dos tecidos dentários, uma vez que evitar o desgaste dentário foi uma preferência relatada pela paciente.

Como etapa preliminar, a contenção semirrígida foi removida com discos polidores (Sof-Lex Pop On, 3M, EUA) no desígnio de se eliminar resquícios do material resinoso na palatina dos dentes superiores e lingual dos inferiores, promovendo a remoção de fatores retentores de biofilme. Um afastador labial com travador de língua (Arcflex, FGM, Brasil) foi colocado e após a profilaxia, a primeira sessão de clareamento de consultório foi iniciada. Para isso, foi utilizada uma barreira de proteção gengival (OpalDam, Ultradent, EUA) fotopolimerizada por 20 segundos (VALO Cordless, Ultradent, EUA), e um gel com peróxido de hidrogênio a $40 \%$, fluoreto de sódio e nitrato de potássio (Opalescence Boost PF, Ultradent, EUA) foi aplicado sobre as superfícies dos dentes por 40 minutos com troca do gel após 20 minutos, de acordo com as especificações do fabricante. O clareamento caseiro foi empregado utilizando um gel contendo peróxido de carbamida a 15\% (Opalescence PF, Ultradent, EUA), o qual foi orientado a ser aplicado dentro dos dispositivos previamente confeccionados. A orientação dada à paciente foi da utilização diária caseira por 1 hora e 30 minutos durante 14 dias, com consultas intercaladas de acompanhamento pelo profissional.

Como relatado previamente, no modelo de gesso foi realizado o enceramento diagnóstico (Figura 2A), etapa indispensável para a avaliação de parâmetros estéticos e oclusais importantes na reabilitação das guias, movimentos de lateralidade, protrusão e retrusão. Assim, foi confeccionada uma matriz/guia em silicone de adição (Express XT, 3M, EUA) para a realização do ensaio restaurador com resina bisacrílica (Protemp 4, 3M, EUA). O mock-up promoveu a visualização e o estudo do sorriso da paciente. Após a aprovação pela mesma (Figura 2B), realizou-se uma nova impressão e obtenção de um novo modelo de gesso para servir como guia do procedimento restaurador.
Após a remoção do mock-up, uma segunda sessão de clareamento dental foi realizada com os mesmos cuidados já descritos, entretanto nessa consulta aplicou-se um gel com peróxido de hidrogênio a 35\% e cálcio (Whiteness HP Blue, FGM, Brasil). A troca do gel clareador foi proposta devido ao relato de sensibilidade da paciente ao final da outra sessão clareadora de consultório.

Após 14 dias da finalização do tratamento clareador, uma melhora significativa da cor foi constatada (Figura 3), e então iniciada a fase restauradora. A confecção de uma matriz/guia de silicone de condensação denso (Zetaplus, Zhermack/Dentsply Sirona, EUA) foi realizada de acordo com o mock-up previamente provado. A seleção das resinas compostas foi realizada através do uso da escala de cor, incrementos na região a ser restaurada e avaliação através de uma foto monocromática (Figura 4). Assim, foram selecionadas as seguintes resinas compostas para estratificação: região palatal (Pearl Smoke, Vit-I-Escence, Ultradent, EUA), dentina (A1D, Forma, Ultradent, EUA), efeito (Ir - B, Vit-I-Escence, Ultradent, EUA) e esmalte (WE, Forma, Ultradent, EUA). O isolamento do campo operatório foi realizado utilizando um afastador bucal (Expandex, Indusbello, Brasil), associado a um sugador de alta potência e roletes de algodão que eram trocados sucessivamente. Iniciou-se o procedimento pela inserção de um fio retrator ( $¥ 000$ Ultrapak, Ultradent, EUA) em cada dente para afastar a gengiva e facilitar a realização das restaurações.

Para a restauração dos dentes 11 e 21, foi utilizada uma fita de isolamento dental (Isotape, TDV, Grupo Septodont, França) nos dentes adjacentes (Figura 5). Nenhum preparo dental foi realizado, apenas a remoção pontual de qualquer material resinoso prévio com uma ponta diamantada (2135FF, Fava, Brasil). Após profilaxia e limpeza da região a ser restaurada, realizou-se o condicionamento com ácido fosfórico a 35\% (Ultra Etch IndiSpense, Ultradent, EUA) por 30 segundos no esmalte e lavagem com água por outros 30 segundos. A região foi seca e o sistema adesivo aplicado (Adper ${ }^{\mathrm{TM}}$ Scotchbond ${ }^{\mathrm{TM}}$ Multiuso, 3M ESPE, EUA) de acordo com as instruções do fabricante, com fotoativação por 20 segundos (VALO Cordless, Ultradent, EUA). Uma matriz de poliéster foi utilizada nos espaços interproximais dos dentes para evitar a união dos elementos dentários durante o procedimento adesivo. 
Com auxílio da matriz/guia (Figura 5B), a estratificação foi iniciada com a reprodução palatal respeitando a anatomia estabelecida pelo enceramento (Figura 5C). Para cada etapa realizada, que será descrita a seguir, foi padronizada uma fotoativação do material restaurador por 20 segundos. A matriz/guia de silicone foi removida e o processo de estratificação foi continuado com uma resina composta de dentina (A1D, Forma) inserida no terço médio e incisal, reproduzindo as características morfológicas dos mamelos (Figura 5D). A resina de efeito foi usada na borda incisal, preenchendo os espaços entre os mamelos. Para auxiliar, um agente umidificador de resina (Composite Wetting Resin, Ultradent, EUA) foi utilizado com ajuda de um pincel pelo de marta ( $n^{\circ} 4$ formato quadrado, Tigre, Peru) para facilitar a adaptação do material. A porção vestibular foi restaurada utilizando um guia comercial (Figura 5E) para confecção de facetas em resinas composta (Uveneer, Ultradent, EUA), para isso uma matriz de poliéster nas faces proximais foi utilizada para evitar a ferulização com os dentes adjacentes. Após a acomodação do dispositivo, uma leve pressão foi feita e a matriz de poliéster foi deslizada em direção palatina para proporcionar um melhor acabamento e adaptação nas margens, e então uma nova fotoativação foi realizada com o dispositivo ainda em posição. Imediatamente após o procedimento, o fio retrator foi removido e os excessos cervicais foram delicadamente retirados com auxílio de uma lâmina de bisturi \#12 (Solidor, Brasil)

Após ajuste oclusal, para o acabamento precedeu-se a confecção das linhas de ângulo para determinar a área de espelho, e com discos abrasivos (Sof-Lex Pop On, 3M, EUA) o desgaste foi realizado, associada à determinação da anatomia e texturização utilizando pontas diamantadas. A etapa de polimento foi iniciada com pontas abrasivas (liffy, Ultradent, EUA) utilizadas em ordem decrescente de granulação, intercaladas por pontas diamantadas de granulação ultra fina (Fava, Brasil) para confeccionar a anatomia vestibular, seguida por uma escova de carbeto de silício (Occlubrush, Kavo/Kerr, EUA) utilizada em baixa pressão, e finalizada com uma pasta de polimento (Diamond Polish Mint, Ultradent, EUA) associada a uma escova de algodão (Dhpro, Brasil) para o brilho final. Foi realizado o mesmo procedimento restaurador nos demais dentes (Figuras $5 \mathrm{~F} \mathrm{e} 5 \mathrm{G}$ ), com exceção do guia comercial de confecção de facetas que não foi utilizado nos incisivos laterais (12 e 22) e pré-molares (14 e 24). A paciente ficou satisfeita com o restabelecimento estético e funcional promovido pelo tratamento (Figura 6) e permanece em acompanhamento.

Figura 1. A: Aspecto inicial do sorriso. B: Aspecto intrabucal inicial dos dentes
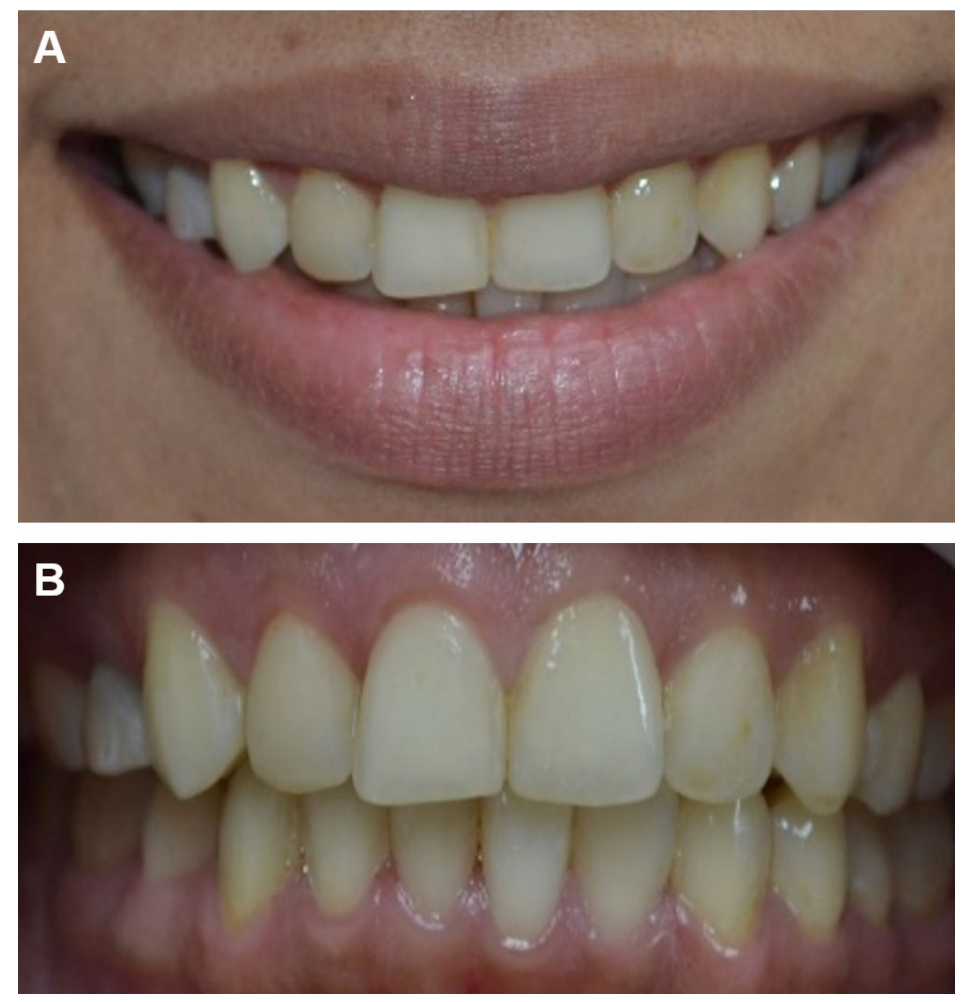
Figura 2. A: Enceramento diagnóstico em articulador semi-ajustável. B: Mock-up em resina bisacrílica
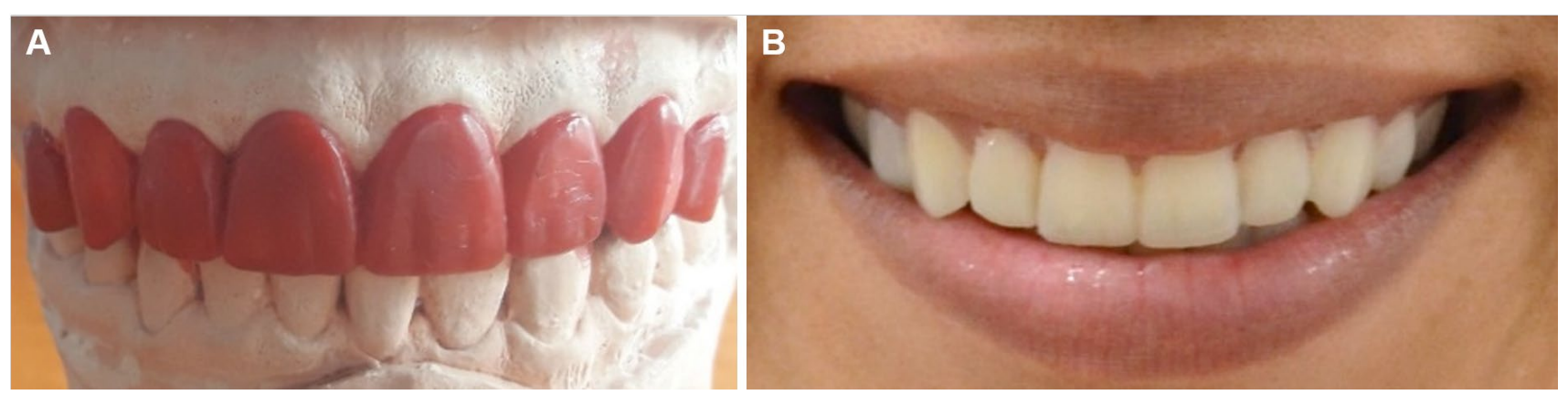

Figura 3. A e B: Aspecto dos dentes e sorriso após clareamento dental realizado pela técnica combinada
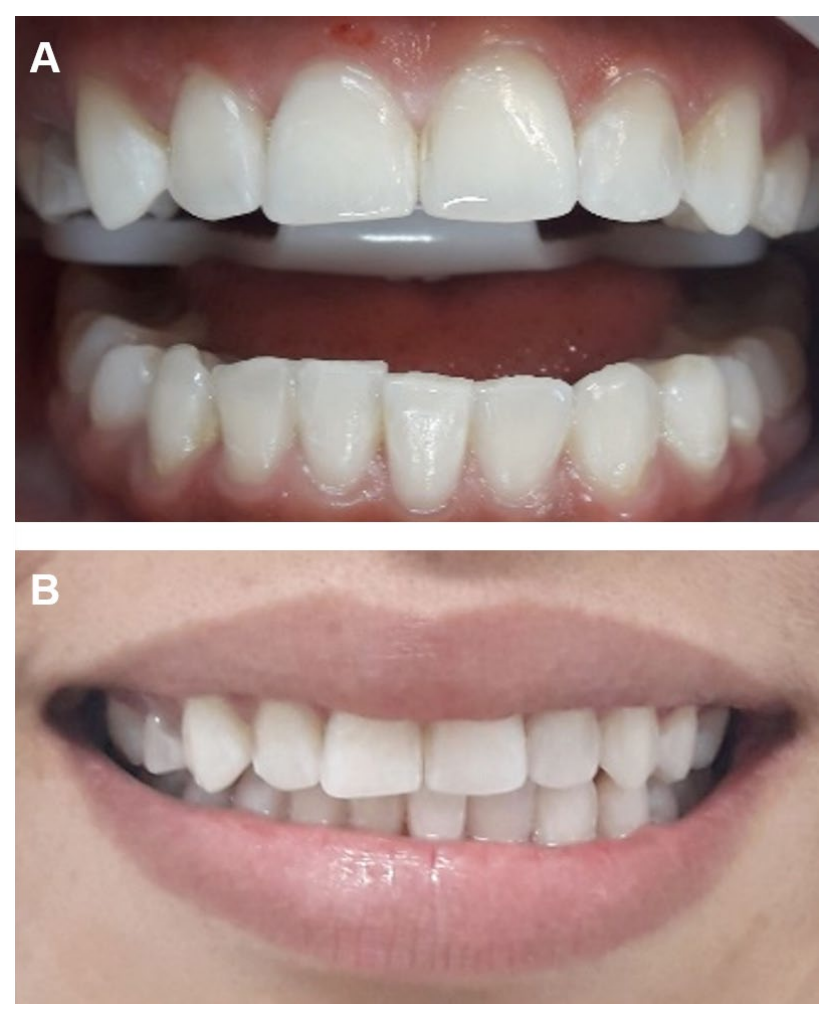
Figura 4. A: Seleção de cor das resinas compostas. B: Foto monocromática para auxiliar na avaliação do valor das resinas compostas
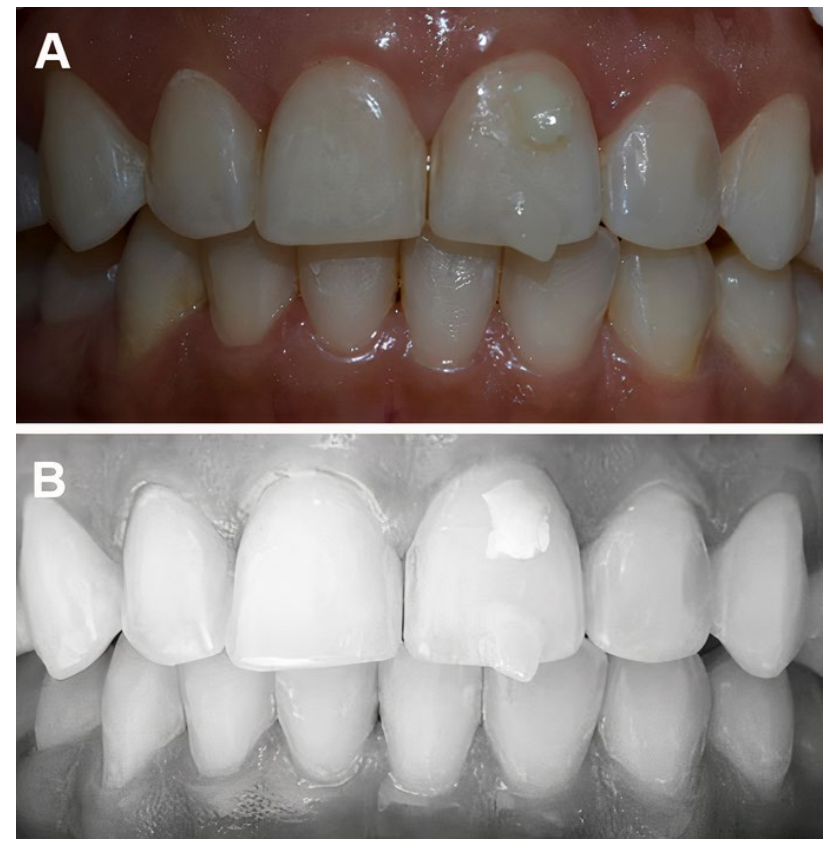

Figura 5. Restaurações diretas em resina composta. A: Inserção de fio retrator e fita de isolamento dental para proteção dos dentes adjacentes. B: Uso de guia para a confecção da concha palatina. C: Reconstrução em resina composta da palatina. D: Inserção da resina composta de dentina. E: Utilização guia comercial para confecção da faceta em resinas composta. F e G: Continuação do procedimento restaurador repetindo as etapas acimas mencionadas nos caninos (13 e 23)
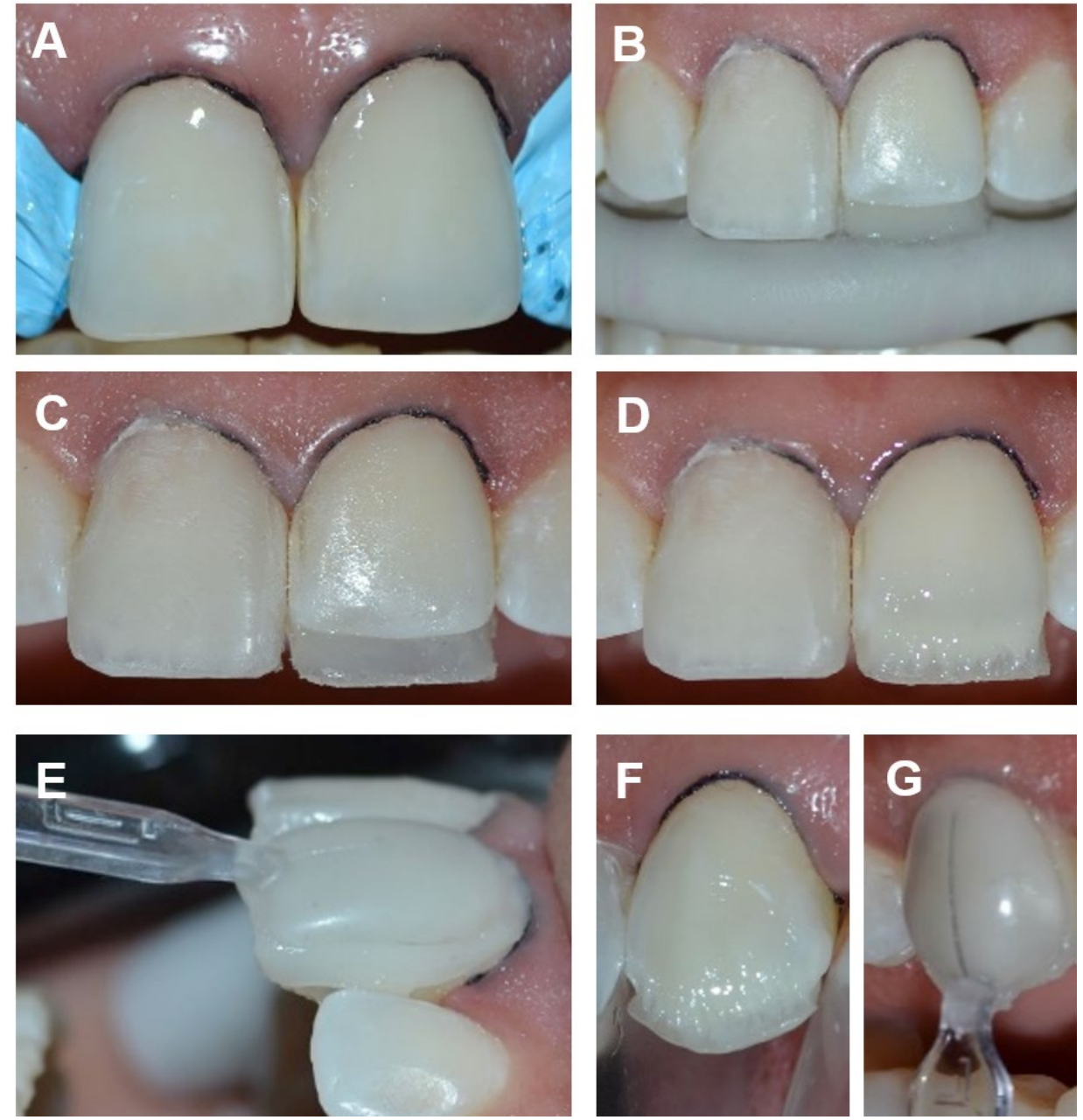

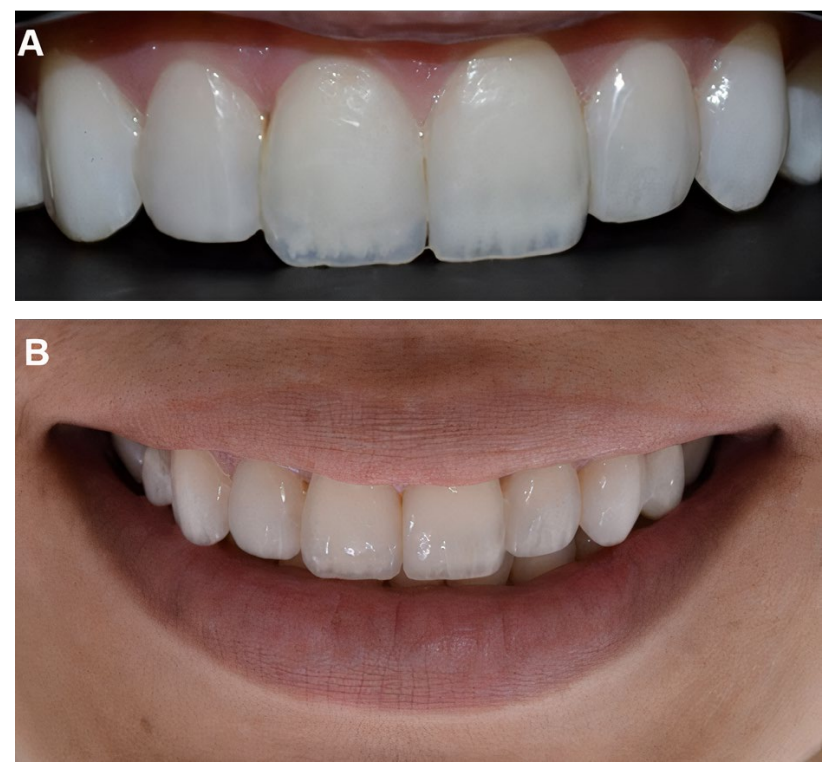

\section{Discussão}

O profissional deve possuir conhecimento dos materiais e a combinação correta dos diferentes tratamentos, preservando a estrutura dental sem causar dano. Diferentes estudos, pesquisas laboratoriais, clínicas e relatos de caso auxiliam na propagação do conhecimento sobre técnicas rotineiras que devem ser embasadas na literatura. O sucesso do tratamento se inicia com um planejamento adequado, exemplificado nesse caso com o estudo da oclusão em articulador semiajustável e da estética do sorriso com o ensaio restaurador (mock-up).

O sorriso do paciente está associado ao conforto psicossocial, autoestima e qualidade de vida, o que exige do profissional a realização de tratamentos adequados e restaurações imperceptíveis ${ }^{9}$. A Odontologia estética consegue promover a melhora da autoestima e autoimagem do indivíduo, associando-se à crescente valorização de um sorriso esteticamente agradável com a busca por diferentes alternativas de tratamento. No presente caso, 0 tratamento foi iniciado pelo procedimento clareador uma vez que a padronização da cor dos substratos favorece a técnica restauradora e mimetização da anatomia dental, além de promover um sorriso mais harmônico com os dentes que não foram restaurados representados pelos dentes inferiores.

O clareamento dental com produtos à base de peróxido de hidrogênio é um processo dinâmico que envolve interações micromorfológicas nos substratos e potencial efeito oxidante sobre as moléculas de pigmentos, mecanismo que modifica as propriedades cromáticas do dente deixando-o mais claro e menos amarelado ${ }^{10}$. Apesar de seguro, alguns efeitos adversos transitórios são associados a esse tratamento, como a sensibilidade dental ${ }^{5}$ e alteração das propriedades físico-químicas dos tecidos dentários ${ }^{14}$. Apesar de o gel utilizado na primeira sessão clareadora ser seguro e validado pela literatura, a troca por outro gel que tinha em sua composição uma menor concentração de peróxido de hidrogênio associado ao gluconato de cálcio foi baseada em um estudo prévio que sugeriu o uso desses géis para diminuir a sensibilidade durante o tratamento clareador de consultório ${ }^{12}$ sem perder a eficácia clareadora ou promover alterações na microdureza do esmalte ${ }^{13}$. No presente caso, a troca dos géis promoveu uma diminuição do relato da sensibilidade após a consulta clínica pela paciente, entretanto esse achado deve ser validado por futuros ensaios clínicos controlados e randomizados. 
O clareamento de dentes vitais pode ser realizado pela técnica de consultório, técnica caseira/domiciliar ou técnica combinada, que é a associação dessas duas técnicas. A técnica combinada tem efetividade clínica estabelecida ${ }^{14}$ corroborando com os resultados do presente caso clínico. Após a finalização do tratamento clareador, um período de 14 dias foi aguardado antes das restaurações em resina composta no intuito de promover uma estabilização de cor e possibilitar um correto processo adesivo. Esse passo deve ser respeitado, visto que o processo de difusão dos agentes clareadores pode promover a formação de oxigênio residual, o que resultaria na diminuição das forças de adesão e inadequada polimerização dos materiais resinosos ${ }^{15}$.

Desde a introdução das resinas compostas no mercado, esses materiais passaram por um desenvolvimento tecnológico considerável, e hoje se apresentam como uma escolha segura para as restaurações parciais dos dentes anteriores. Restaurações diretas em resina composta são alternativas para um tratamento estético e conservador, com efetividade e durabilidade sugerida por relatos de caso e pesquisas de acompanhamento clínico ${ }^{5,16}$. A versatilidade desse material é associada à possibilidade de reparo e realização do procedimento com mínimo desgaste ou, até mesmo, com ausência de preparo do elemento dentário, permitindo a manutenção das estruturas pelo princípio da Odontologia minimamente invasiva.

As resinas compostas escolhidas para a realização das restaurações no presente caso clínico foram uma micro-híbrida (Vit-I-Escence, Ultradent) de efeito para auxiliar no mimetismo das propriedades ópticas do dente, e para simular a estrutura e superfície do dente foi utilizada uma resina nano-híbrida (Forma, Ultradent). Compósitos nano-híbridos apresentam boas propriedades mecânicas e ópticas, além de comportamento clínico adequado17,18. Em especial, a resina composta utilizada é um sistema comercializado numa variedade de cores, o que facilita a técnica estratificada.

Tratamentos estéticos e funcionais como o apresentado no presente trabalho são cada vez mais requisitados na rotina do consultório, entretanto, antes da popularização ou indicação massiva de procedimentos dessa natureza, o profissional precisa compreender que a aplicação inadequada das técnicas e manipulação incorreta dos materiais podem ocasionar injúrias aos tecidos dentários ou resultados estéticos artificiais ${ }^{5-7}$. O operador precisa sistematizar todos os processos relacionados ao planejamento, indicações, técnica adesiva e fotopolimerizadora, polimento/acabamento da restauração e estudo da cor, incluindo a inter-relação da estrutura natural do dente com os materiais restauradores. Imperícias durante o procedimento podem ocasionar restaurações com acabamento e polimento inadequado, acúmulo de biofilme, irritação gengival, manchamentos do material, falha adesiva e, consequentemente, insucesso do tratamento.

O domínio das diferentes técnicas exemplificadas nesse caso clínico como a indicação racional dos procedimentos restauradores diretos, conhecimento dos princípios da adesão e a manipulação correta dos materiais são importantes no sucesso de procedimentos estéticos e restauradores, onde o objetivo final deve ser um resultado funcional, natural, estético e que acima de tudo preserve a saúde dos tecidos orais.

\section{Conclusão}

O tratamento restaurador direto utilizando resinas compostas após o clareamento dental realizado pela técnica combinada promoveu o restabelecimento estético e funcional do sorriso, através de uma abordagem conservadora.

\section{Contribuições dos autores}

Vargas-Mendoza MJ e Vieira-Junior WF participaram da concepção do estudo, do relato e análise do caso, da escrita do manuscrito e da aprovação final. Junqueira AMR, Telles CCC e Carnaúba GRP participaram da concepção do estudo, do relato, da escrita do manuscrito e da aprovação final.

\section{Conflitos de interesses}

Nenhum conflito financeiro, legal ou político envolvendo terceiros (governo, empresas e fundações privadas, etc.) foi declarado para nenhum aspecto do trabalho submetido (incluindo, mas não se limitando a subvenções e financiamentos, participação em conselho consultivo, desenho de estudo, preparação de manuscrito, análise estatística, etc.). 


\section{Referências}

1. Wirsching E. Contemporary options for restoration of anterior teeth with composite. Quintessence Int. 2015;46(6):457-63. http:// dx.doi.org/10.3290/j.qi.a33989

2. Di Murro B, Gallusi G, Nardi R, Libonati A, Angotti V, Campanella $V$. The relationship of tooth shade and skin tone and its influence on the smile attractiveness. J Esthet Restor Dent. 2020;32(1):57-63. http://dx.doi.org/10.1111/jerd.12543

3. Vieira-Junior WF, Sugii MM, Theobaldo JD, Paulillo LAMS, Lovadino JR, Aguiar FHB et al. Resolução estética de um caso de fluorose através de clareamento dental: relato de caso clínico. Arch Health Invest. 2015;4(5):41-5.

4. Geus JL, Wambier LM, Kossatz S, Loguercio AD, Reis A. At-home vs In-office Bleaching: A Systematic Review and Meta-analysis. Oper Dent. 2016;41(4):341-56. http://dx.doi.org/10.2341/15-287LIT

5. Rezende M, Loguercio AD, Kossatz S, Reis A. Predictive factors on the efficacy and risk/intensity of tooth sensitivity of dental bleaching: A multi regression and logistic analysis. J Dent. 2016;45:1-6. http://dx.doi.org/10.1016/j.jdent.2015.11.003

6. Korkut B, Yanıkoğlu F, Günday M. Direct composite laminate veneers: three case reports. J Dent Res Dent Clin Dent Prospects. 2013;7(2):105-11. http://dx.doi.org/10.5681/joddd.2013.019

7. Dietschi D, Shahidi C, Krejci I. Clinical performance of direct anterior composite restorations: a systematic literature review and critical appraisal. Int J Esthet Dent. 2019;14(3):252-70.

8. Fahl Júnior $\mathrm{N}$. The direct/indirect composite resin veneers: a case report. Pract Periodontics Aesthet Dent. 1996;8(7):627-38.

9. Maia TS, Borges MG, Silva FP, Menezes MS. Harmonização do sorriso com facetas diretas em resina composta: relato de caso. Clín Int J Braz Dent. 2015;11(4):392-401.

10. Kwon SR, Wertz PW. Review of the Mechanism of Tooth Whitening. J Esthet Restor Dent. 2015;27(5):240-57. http://dx.doi. org/10.1111/jerd.12152
11. Alqahtani MQ. Tooth-bleaching procedures and their controversial effects: A literature review. Saudi Dent J. 2014;26(2):33-46. http://dx.doi.org/10.1016/j.sdentj.2014.02.00215

12. Kossatz S, Martins G, Loguercio AD, Reis A. Tooth sensitivity and bleaching effectiveness associated with use of a calcium-containing in-office bleaching gel. J Am Dent Assoc. 2012;143(12):81-7. http://dx.doi.org/10.14219/jada. archive.2012.007516

13. Vieira I, Vieira-Junior WF, Pauli MC, Theobaldo JD, Aguiar FHB, Lima DANL et al. Effect of in-office bleaching gels with calcium or fluoride on color, roughness, and enamel microhardness. J Clin Exp Dent. 2020;12(2):116-22. http://dx.doi.org/10.4317/jced.56006

14. Dawson PFL, Sharif MO, Smith AB, Brunton PA. A clinical study comparing the efficacy and sensitivity of home vs combined whitening. Oper Dent. 2011;36(5):460-6. http://dx.doi. org/10.2341/10-159-C

15. Yu H, Zhang CY, Cheng SL, Cheng H. Effects of bleaching agents on dental restorative materials: A review of the literature and recommendation to dental practitioners and researchers. J Dent Sci. 2015;10(4): 345-51. http://dx.doi.org/10.1016/j. jds.2014.08.005

16. Gouveia THN, Theobaldo JD, Vieira-Junior WF, Lima DANL, Aguiar FHB. Esthetic smile rehabilitation of anterior teeth by treatment with biomimetic restorative materials: a case report. Clin Cosmet Investig Dent. 2017;9:27-31. http://dx.doi. org/10.2147/CCIDE.S130698

17. Angerame D, De Biasi M. Do Nanofilled/Nanohybrid Composites Allow for Better Clinical Performance of Direct Restorations Than Traditional Microhybrid Composites? A Systematic Review. Oper Dent. 2018; 43(4):191-209. http://dx.doi. org/10.2341/17-212-L

18. Maran BM, Geus JL, Gutiérrez MF, Heintze S, Tardem C, Barceleiro MO et al. Nanofilled/nanohybrid and hybrid resin-based composite in patients with direct restorations in posterior teeth: A systematic review and meta-analysis. J Dent. 2020;99:103407. http://dx.doi.org/10.1016/j.jdent.2020.103407 